\title{
Aggravated intestinal ischemia-reperfusion injury is associated with activated mitochondrial autophagy in a mouse model of diabetes
}

\author{
ZI ZENG ${ }^{*}$, HUI-MIN LIU*, YUN-YAN ZHANG, RONG CHEN, TAO SUN, WEI LI, \\ QIAN SUN, ZHONG-YUAN XIA and QING-TAO MENG
}

Department of Anesthesiology, Renmin Hospital of Wuhan University, Wuhan, Hubei 430060, P.R. China

Received August 14, 2019; Accepted April 6, 2020

DOI: $10.3892 / \mathrm{mmr} .2020 .11270$

\begin{abstract}
Numerous studies have reported that diabetes is associated with an increased susceptibility to cardiac ischemiareperfusion injury; however, the mechanism underlying the role of diabetes during intestinal ischemia-reperfusion (IIR) has yet to be elucidated. The present study evaluated the intestinal pathological alterations and possible underlying mechanisms in a mouse model of type 1 diabetes mellitus with IIR. The effects of diabetes were investigated by assessing the histopathology, oxidative stress, inflammatory cytokine levels in intestine tissues and blood plasma, and protein expression levels of phosphatase and tensin homolog-induced putative kinase (PINK1), Parkin and the ratio of light chain 3B (LC3B) II/I. The results demonstrated that diabetes increased the Chiu's intestinal injury score, concentration of interleukin (IL)-1 $\beta$, IL- 6 and tumor necrosis factor (TNF)- $\alpha$, and levels of oxidative stress. Furthermore, the alterations were more pronounced in the diabetes with IIR group. The expression levels of PINK1 and Parkin, as well as the ratio of LC3BII/I, were significantly upregulated in the IIR group compared with the Sham group. Diabetes activated PINK1 and Parkin, and increased the expression of LC3BII. Furthermore, transmission electron microscopy revealed that mitochondrial destruction and the number of autophagosomes was increased in the diabetic groups compared with the non-diabetic groups. Collectively, the results of the present study suggest that diabetes increased intestinal vulnerability to IIR by enhancing inflammation and oxidative stress. Furthermore, IIR was associated with overactivation of mitochondrial autophagy; therefore, the
\end{abstract}

Correspondence to: Professor Qing-Tao Meng, Department of Anesthesiology, Renmin Hospital of Wuhan University, 238 Jiefang Road, Wuhan, Hubei 430060, P.R. China

E-mail: mengqingtao2018@126.com

*Contributed equally

Key words: intestinal ischemia-reperfusion injury, diabetes, mitochondrial autophagy increased vulnerability to IIR-induced intestine damage due to diabetes may be associated with PINK1/Parkin-regulated mitochondrial autophagy.

\section{Introduction}

Diabetes increases sensitivity to ischemia-reperfusion injury, and may be associated with increased levels of oxidative stress during hyperglycemia (1). The increased oxidative stress in diabetes is primarily caused by an imbalance between the generation and elimination of oxidative stress. Reactive oxygen species (ROS) are associated with the endogenous antioxidant defense system (2). In addition, due to elevated levels of blood glucose, osmotic dehydration is observed in the mouse model of diabetes, which leads to increased permeability of the intestinal cells, an impaired normal mucosal barrier of the small intestine and increased intestinal damage (3).

Intestinal ischemia reperfusion (IIR) injury is a clinical challenge associated with high morbidity and mortality, which has important roles during numerous pathological processes, including neonatal necrotizing enterocolitis (4), acute mesenteric ischemia (5), intestinal torsion (6), bowel transplantation (7), trauma (8), shock $(8,9)$, and cardiopulmonary insufficiency (10). Ischemic injury is primarily caused by interrupted blood flow triggered by hypoxia, which leads to the death of intestinal mucosa cells and severe mucosal damage (11). IIR occurs when blood flow is restored, causing further damage to the viable mucosal cells and resulting in the release of various inflammatory mediators, which potentially leads to systemic inflammatory response syndrome and ultimately to multiple organ failure (12). The pathophysiological mechanism underlying IIR has yet to be fully elucidated; however, considerable evidence has indicated that IIR-induced injury is associated with the appearance of inflammatory mediators (including ROS, cytokines and bacterial endotoxins), impaired mitochondrial function and apoptosis (13-16).

Previous studies have reported that diabetes is associated with increased susceptibility to cardiac ischemia-reperfusion injury $(1,17)$, which may be associated with increased levels of underlying oxidative stress that are secondary to hyperglycemia $(18,19)$. The increase in oxidative stress that occurs during diabetes is primarily due to the imbalance between the 
production of reactive oxygen species (ROS) and the removal of the endogenous antioxidant defense system. The degree of imbalance may affect the severity of ischemia-reperfusion injury under diabetic conditions (20). During diabetes, mitochondria are the major intracellular sources of ROS, and $\sim 4 \%$ of the oxygen consumed by mitochondria is converted into ROS (21). Mitochondria are the main targets of oxidative damage and damaged mitochondria can further increase ROS production via ROS-induced release of ROS (22), which may lead to leakage of lethal factors such as interleukin (IL)- $1 \beta$ and IL-6 (23), triggering a vicious cycle which induces myocardial cell death (24). Diabetes is at least partly considered to be a mitochondrial disease, since the overproduction of superoxide in the mitochondrial electron transport chain is involved in the mechanism underlying hyperglycemia-induced injury $(25,26)$. A previous study on diabetic ischemic reperfusion of the myocardium revealed that decreased levels of mitochondrial autophagy promote mitochondrial aggregation in the myocardium, aggravate mitochondrial disturbances in myocardial tissue, damage myocardial function and increase mortality in a mouse model of diabetes (27). The discovery of autophagy during ischemia-reperfusion provides a novel therapeutic target for ischemic heart disease; however, only a few studies have reported the effects of mitochondrial autophagy during diabetic IIR. Therefore, the present study aimed to assess the effect of diabetes on intestinal pathological alterations in a mouse model of type 1 diabetes mellitis with IIR.

\section{Materials and methods}

Animals. The present study was approved by the Animal Care Committee of Wuhan University and protocols were conducted in accordance with the National Institutes of Health Guidelines for the Care and Use of Experimental Animals (28). A total of 40 healthy wild-type SPF male C57BL/6J mice (age, 6-8 weeks; weight, 20-25 g) were purchased from Beijing Weitong Lihua Experimental Animal Technology Co., Ltd. All animals were housed in individual cages $(\mathrm{n}=2 / 3$ per cage) in a climate-controlled room $\left(23 \pm 1^{\circ} \mathrm{C}\right.$; relative humidity, $\left.60 \pm 5 \%\right)$ with 12-h light/dark cycles and free access to food and water for one week. All mice were fasted with free access to water for $12 \mathrm{~h}$ prior to the experiments.

Diabetes model. All mice were fasted with free access to water for $12 \mathrm{~h}$ prior to the experiments. After a 72-h fast, the diabetic group were administrated streptozocin $(220 \mathrm{mg} / \mathrm{kg}$; Sigma-Aldrich; Merck KGaA) intraperitoneally. After $72 \mathrm{~h}$, the blood glucose level in the tail blood was tested. Symptoms of polydipsia, polyphagia and diabetes indicated successful induction of the mouse model of diabetes. During the experimental period ( 2 months), blood was collected every week to monitor blood glucose levels. If the blood glucose level was $\geq 16.7 \mathrm{mmol} / 1$ for three consecutive weeks, the mouse model of diabetes was considered to have been successfully established. The non-diabetic group received an equal volume of saline $(200 \mathrm{mg} / \mathrm{kg})$.

IIR model. The IIR model was established according to the protocol previously described by Meng et al (29). Mice were anesthetized by the intraperitoneal injection of pentobarbital sodium solution $(1 \% ; 40 \mathrm{mg} / \mathrm{kg})$ and were subsequently fixed in the supine position. The fur in the abdominal region was shaved and the area was disinfected. A midline incision was made and the roots of the superior mesenteric artery (SMA) were clamped using micro-artery clamps. Subsequently, vascular contractions were reduced, the intestinal wall became pale and the mesentery was observed. After the arterial blood flow was blocked, the wound was closed. After $45 \mathrm{~min}$, the arterial clamp was released to restore blood supply, modeling IIR. The Sham group and DS groups were subjected to the same procedure; however, the vessels were not clamped. Mice were sacrificed $2 \mathrm{~h}$ post-ischemia-reperfusion clamping. The blood and intestine tissues were collected and processed for subsequent biochemical analysis. The small intestine tissue was isolated, washed with saline, fixed with $4 \%$ paraformaldehyde at $4^{\circ} \mathrm{C}$ for $24-48 \mathrm{~h}$ and frozen in liquid nitrogen.

Experimental grouping. Following surgical preparation, the mice were randomly divided into four groups ( $\mathrm{n}=10$ per group): i) Control group (Sham group); ii) normal IIR group (IIR group); iii) diabetic non-ischemic reperfusion group (DS group); and iv) diabetic ischemia-reperfusion group (DIIR group).

Histopathological assessment of the intestines. The fresh ischemic area of the intestine was washed three times in cold phosphate buffer ( $\mathrm{pH} 7.4)$, fixed in $4 \%$ formaldehyde $(\mathrm{pH} 7.4)$ at $4^{\circ} \mathrm{C}$ for $24-48 \mathrm{~h}$, paraffin-embedded and sectioned at a thickness of $5 \mu \mathrm{m}$. Sections were then stained in Harris hematoxylin solution for 5-20 min and with eosin solution (95\% ethanol solution) for 3-30 sec at room temperature. Stained sections were observed using an Olympus BX50 (Olympus Corporation) light microscope at $\mathrm{x} 200$ magnification. The HPIAS-1000 medical color image analysis system (Olympus Corporation) was used to collect images and Image Pro Plus software (version 6.0; Media Cybernetics, Inc.) was used for analysis. For each specimen, two sections were observed (10 fields of view), intestinal pathological alterations were identified and Chiu's pathology scores were calculated (25). Higher scores indicated more severe damage. The Chiu grading system consists of 5 subdivisions according to alterations to the villus and gland of the intestinal mucosa: Grade 0 , normal mucosa; grade 1, development of subepithelial Gruenhagen's space at the tip of the villus; grade 2 , extension of the space with moderate epithelial lifting; grade 3, massive epithelial lifting with a few denuded villi; grade 4, denuded villi with exposed capillaries; and grade 5, disintegration of the lamina propria, ulceration and hemorrhage.

Measurement of malondialdehyde (MDA). Following homogenization of the tissue in normal saline on ice or centrifugation at $13,000 \times \mathrm{g}$ for $10 \mathrm{~min}$ at $4^{\circ} \mathrm{C}$ of the blood, the MDA levels of the supernatants were determined using an MDA assay kit (cat. no. A001-3-2; Nanjing Jiancheng Bioengineering Institute) according to the manufacturer's protocol and the thiobarbituric acid colorimetric method, as previously described (30). MDA concentration is expressed as $\mathrm{nmol} / \mathrm{mg}$ protein.

Activity of superoxide dismutase (SOD) and glutathione peroxidase $(G S H-P x)$ in intestinal tissues. SOD activity was measured using the SOD assay kit (Nanjing Jiancheng 
Bioengineering Institute) according to the manufacturer's protocol and calculated according to the following method. Briefly, epinephrine undergoes autoxidation rapidly at $\mathrm{pH} 10.0$ to produce adrenochrome, a pink-colored product that can be detected at a wavelength of $480 \mathrm{~nm}$ using a UV-vis spectrophotometer in the kinetic mode (31). The amount of enzyme required to produce $50 \%$ inhibition was defined as one unit of enzyme activity. SOD activity is expressed as units/mg protein.

GSH-Px activity was measured using the GSH-Px assay kit (Nanjing Jiancheng Bioengineering Institute), according to the manufacturer's protocol. The reaction was initiated by the addition of $\mathrm{H}_{2} \mathrm{O}_{2}$. A series of enzymatic reactions were activated by GSH-Px in the homogenate that subsequently led to the conversion of reduced glutathione (GSH) into oxidized glutathione (GSSG). Alterations to the absorbance at a wavelength of $340 \mathrm{~nm}$ during the conversion of GSH to GSSG were recorded using a spectrophotometer. Enzyme activity was calculated according to a formula provided by the manufacturer. GSH-Px activity is expressed as $\mathrm{nmol} / \mathrm{mg}$ protein.

TNF- $\alpha, I L-6, I L-10$ and IL-1 $\beta$ levels in the blood plasma and intestine tissue. The inflammatory cytokine levels of TNF- $\alpha$, IL-6 and IL-10 in the blood plasma and intestinal tissues were quantified using ELISA kits (32). The following kits were used according to the manufacturer's protocol: TNF- $\alpha$ ELISA kit (cat. no. H052; Nanjing Jiancheng Bioengineering Institute), IL-1 $\beta$ ELISA kit (cat. no. H002; Nanjing Jiancheng Bioengineering Institute) and IL-6 ELISA kit (cat. no. H007; Nanjing Jiancheng Bioengineering Institute). Cytokine levels are expressed as $\mathrm{pg} / \mathrm{ml}$.

Western blot analysis. The intestines were isolated and washed three times with pre-chilled PBS at $4^{\circ} \mathrm{C}$. Subsequently, total protein was extracted from the intestinal tissues using RIPA lysate (cat. no. MT006; Biolab Technology) on ice for $30 \mathrm{~min}$. Cell suspensions were lysed by sonification at $400 \mathrm{w}$ on crushed ice every $10 \mathrm{sec} 15-20$ times and then centrifuged at $4^{\circ} \mathrm{C}$ for $15 \mathrm{~min}$ at $12,000 \mathrm{x} \mathrm{g}$. Total protein was quantified using a bicinchoninic acid assay and subsequently, $5 \mathrm{X}$ loading buffer (BioMart) was added to the samples and boiled for $10 \mathrm{~min}$. Equal amounts of protein $(50 \mu \mathrm{g})$ were separated via $12 \%$ SDS-PAGE at $100 \mathrm{~V}$ for $3 \mathrm{~h}$ and transferred onto PVDF membranes. The membranes were blocked with $5 \%$ fat-free milk powder for $1 \mathrm{~h}$ at room temperature. Subsequently, the membranes were incubated overnight at $4^{\circ} \mathrm{C}$ in a shaker with rabbit anti-mice polyclonal primary antibodies targeted against: Parkin (cat. no. 2132; 1:1,000; Cell Signaling Technology, Inc.), Pink1 (cat. no. ab137361; 1:1,000; Abcam), LC3B (cat. no. ab48394; 1:1,000; Abcam) and $\beta$-actin (cat. no. 4970; 1:2,000; Cell Signaling Technology, Inc.). Subsequently, the membranes were washed three times with TBST at room temperature for $10 \mathrm{~min}$ per wash. Following primary incubation, the membranes were incubated with a LI-COR IRDye800CW-conjugated goat anti-rabbit secondary antibody (1:10,000; cat. no. 926-32211; LI-COR Biosciences) for $1 \mathrm{~h}$ at room temperature. The membranes were washed three times with TBST at room temperature. Protein bands were visualized and using the ODYSSEY two-color infrared laser imaging system (LI-COR Biosciences). Protein expression was quantified using Odyssey software (version 3.0, LI-COR Biosciences) using $\beta$-actin as the loading control.

Transmission electron microscopy (TEM). Intestinal tissue samples were pre-fixed with $4 \%$ glutaraldehyde for $2 \mathrm{~h}$ at $4{ }^{\circ} \mathrm{C}$ and subsequently fixed with $2 \%$ osmium tetroxide for $2 \mathrm{~h}$ at $20^{\circ} \mathrm{C}$. Following washing with PBS and dehydration using a graded ethanol series, tissue samples were embedded in epoxy-resin for $48 \mathrm{~h}$ at room temperature. Ultrathin sections $(60-80 \mathrm{~nm})$ were cut using an Ultracut UCT ultramicrotome (Leica Microsystems $\mathrm{GmbH}$ ). Samples were stained with lead citrate for $30 \mathrm{~min}$ at room temperature. The ultrastructure of the tissue sections was observed using a H-7000 FA transmission electron microscope (Hitachi, Ltd.) at x2,000 magnification. Autophagosomes or autophagolysosomes were identified by the characteristic structure of a bilayer or multilayer smooth membrane that completely surrounded the compressed mitochondria or membrane-bound electron-dense material.

Statistical analysis. Data are expressed as the mean \pm SD. Statistical analyses were performed using GraphPad Prism software (version 5.0; GraphPad Software Inc.). Statistical evaluation of the data was performed by two-way ANOVA followed by Tukey's post hoc test. $\mathrm{P}<0.05$ was considered to indicate a statistically significant difference.

\section{Results}

Diabetes increases the intestinal vulnerability to IIR injury. To investigate the effects of diabetes on IIR injury, H\&E staining was performed and Chiu's pathology scores were calculated for the four experimental groups of mice. The intestinal mucosa of the Sham group was intact and the villi of the small intestine were well-arranged (Fig. 1A). In the DS group, the intestinal mucosa was thickened, the small intestine villous epithelial cells were edematous, and a cystic space was observed under the apical epithelium (Fig. 1A). In the IIR group, the intestinal mucosa lost its normal structure, the villi were broken and shed, the interstitium was broken, inflammatory cells had infiltrated and the cellular components of the lamina propria were increased (Fig. 1A). In the DIIR group, destruction of the intestinal mucosa was even more severe compared with the IIR group: The epithelial cell layer was necrotic, and in the process of being shed; the innermost layer was destroyed; and scattered bleeding and ulceration lesions were observed (Fig. 1A). Chiu's pathological scoring system indicated that the degree of intestinal mucosal damage in the DS group was more extensive compared with the Sham group, and the degree of intestinal mucosal injury in the DIIR group was more extensive compared with the IIR group. The degree of injury of the intestinal mucosa in the IIR and DIIR groups was more extensive compared with the Sham and DS groups, respectively. The aforementioned differences were statistically significant $(\mathrm{P}<0.05$; Fig. $1 \mathrm{~A}$ and $\mathrm{B})$.

Diabetes increases the levels of the inflammatory cytokines, $I L-1 \beta, I L-6$ and $T N F-\alpha$, in the blood plasma and intestinal tissue. The levels of IL-1 $\beta$, IL- 6 and TNF- $\alpha$ in the blood 


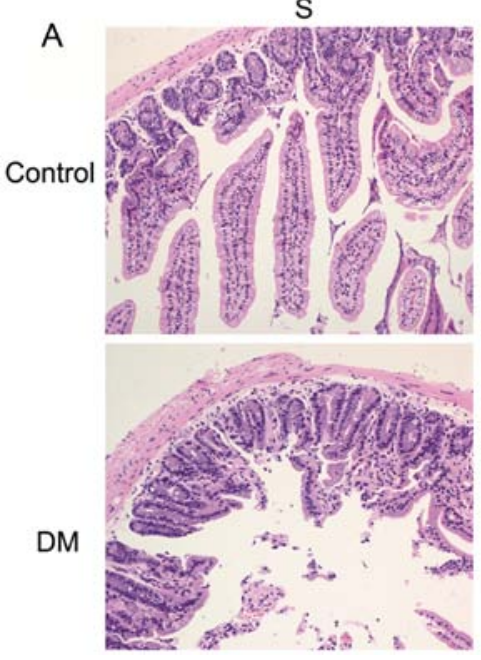

IIR

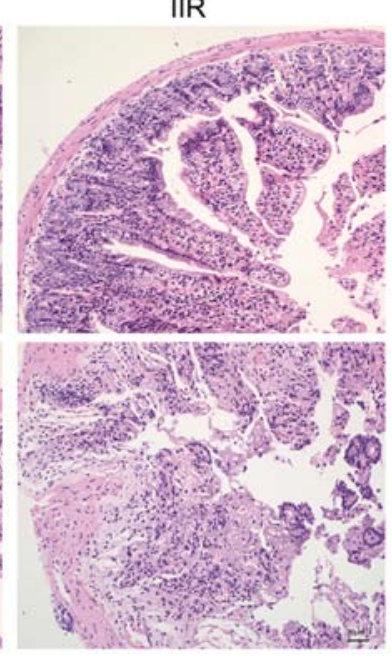

B

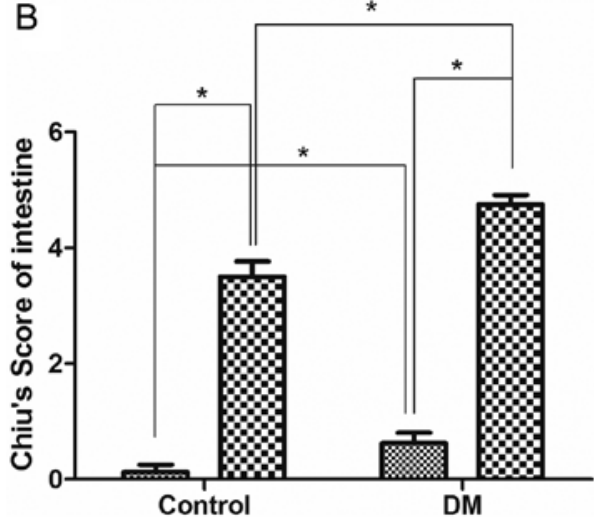

compared with the Sham and IIR groups, respectively $(\mathrm{P}<0.05$; Fig. 3C and D).

Mitochondrial autophagy is significantly increased following IIR in the mouse model of diabetes. Subsequently, TEM was performed to examine the intestinal tissue specimens of the different groups. The DS group displayed mitochondrial swelling, structural integrity and increased numbers of autophagosomes compared with the Sham group (Fig. 4A and B). In the IIR group, mitochondrial swelling was evident, the iliac crest had ruptured, matrix density was decreased, mitochondria were vacuolated, and the number of autophagosomes was increased compared with that of the sham group (Fig. 4A and B). In the DIIR group, a large number of mitochondria were destroyed, the mitochondrial structure was undefined and the number of autophagosomes was significantly increased compared to those of the sham group (Fig. 4A and B).

Subsequently, the expression of the autophagy marker LC3BII/I in intestinal tissues was detected by western blotting. The levels of LC3BII/I were upregulated in the DS and DIIR groups compared with the Sham and IIR groups, respectively. Treatment with IIR significantly increased LC3BII/I levels in the IIR and DIIR groups compared with the Sham and DS groups, respectively. Additionally, the increase in LC3BII/I expression in the DIIR group compared with the DS group was more obvious compared with the increase in the IIR group compared with the Sham group (Fig. 5A and C).

Protein expression of PINK1/Parkin is upregulated following IIR in the mouse model of diabetes. The protein expression levels of PINK1/Parkin were investigated following treatment with IIR by western blotting. The results demonstrated that PINK1/Parkin expression levels were increased in the DIIR and IIR groups compared with the DS and Sham groups, respectively $(\mathrm{P}<0.05$; Fig. $5 \mathrm{~B})$. Furthermore, the increase in expression in the DIIR group compared with the IIR group was more pronounced compared with the increase in expression in the DS group compared with the Sham group (Fig. 5B).

\section{Discussion}

In the present study, the results suggested that diabetes enhanced intestinal damage following IIR, decreased intestinal SOD and GSH-Px activity, and intensified the systemic inflammatory response in mice. Of note, intestinal damage was accompanied by an increase in mitochondrial autophagy in the intestinal tissues, and diabetes further increased IIR-induced increases in PINK1 and Parkin expression, as well as the ratio of LC3BII/I.

Intestinal tissue is sensitive to inflammation due to its anatomical structure, which possesses a large surface area and strong permeability (33). Due to high oxygen demand, the intestine is an ischemia-reperfusion injury sensitive organ. The intestinal mucosal response to ischemia can be divided into two phases: i) During hypoxia, hypoxia causes death of intestinal mucosa cells and severe mucosal damage; and ii) during reperfusion, re-oxidation further damages viable mucosal cells (34). The primary indicator of ischemic injury of the intestinal mucosa is degeneration of the villous epithelium and desquamation of the epithelial layer.

Fig. 3A and B). By contrast, the DS and DIIR groups showe significantly reduced levels of SOD and GSH-Px activity 

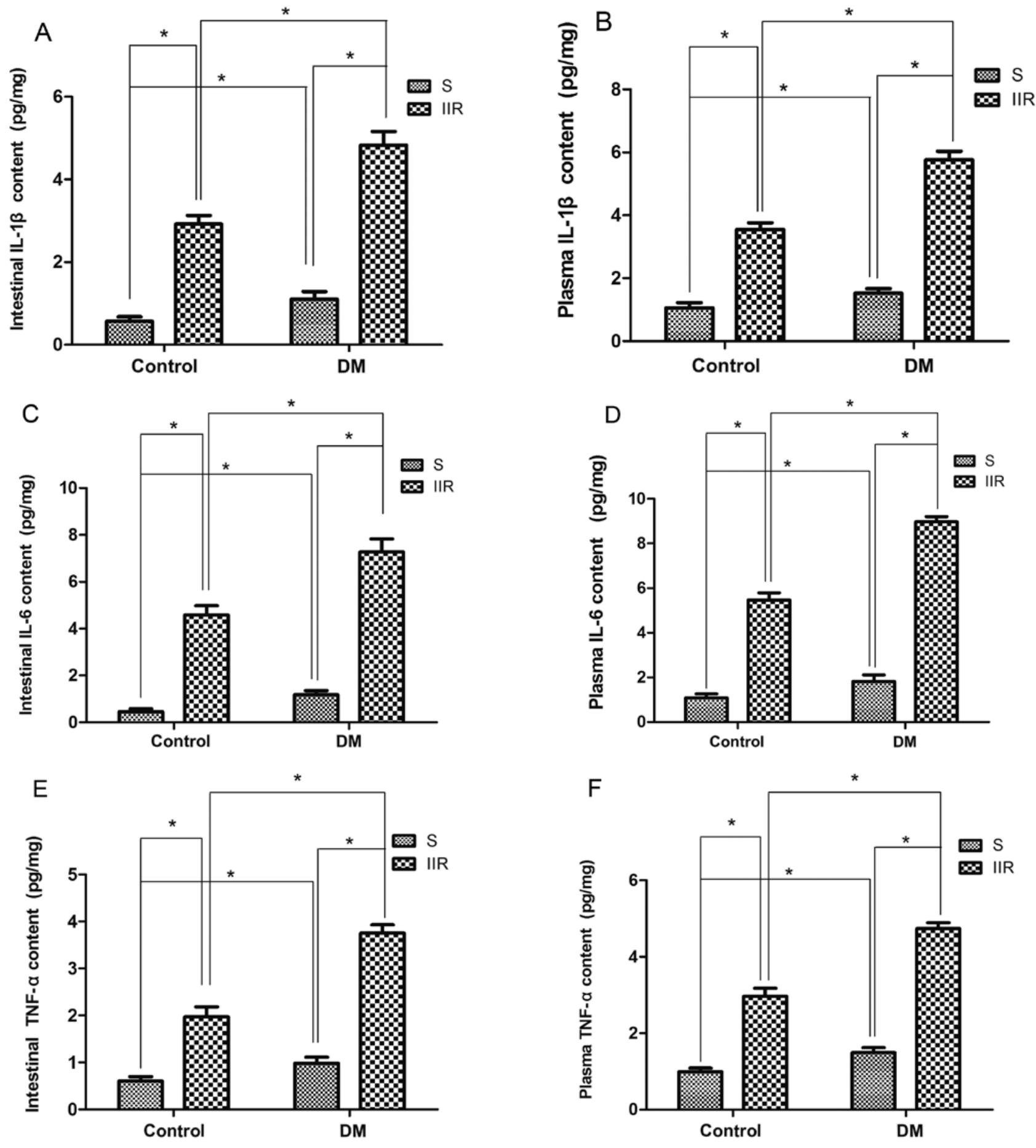

Figure 2. Levels of inflammatory cytokines in the plasma and intestinal tissues were measured using ELISAs. IL-1 $\beta$ content in the (A) intestinal tissues and (B) plasma was measured. IL-6 content in the (C) intestinal tissues and (D) plasma was measured. TNF- $\alpha$ content in the (E) intestinal tissues and (F) plasma was measured. "P $<0.05$, as indicated. IL, interleukin; TNF, tumor necrosis factor; S, Sham; IIR, intestinal ischaemia reperfusion; DM, diabetic mice.

During reperfusion, the villi and mucous membranes are severely damaged due to the production of ROS (34). The main mechanism that triggers ischemia-reperfusion damage is oxidative stress (35). During ischemia-reperfusion injury, oxidative stress is followed by inflammatory cell activation, production of systemic inflammatory mediators, increased bacterial translocation, release of bacterial products including endotoxins, activation of the systemic inflammatory response cascade and multiple organ failure (36). In a mouse model of diabetes, the mitochondria are the major source of ROS in the cell, but the mitochondria are also the main target of oxidative damage (37). Damaged mitochondria further increase ROS production via ROS-induced ROS release (22), resulting in leakage of lethal factors such as IL-1 $\beta$ and IL-6, which triggers a vicious cycle to induce cell death $(23,24)$. In addition, due to elevated blood glucose, osmotic dehydration is often observed in the mouse model of diabetes, which leads to increases in intestinal permeability, an impaired normal mucosal barrier of the small intestine and increased intestinal damage (3). Therefore, it was hypothesized that 

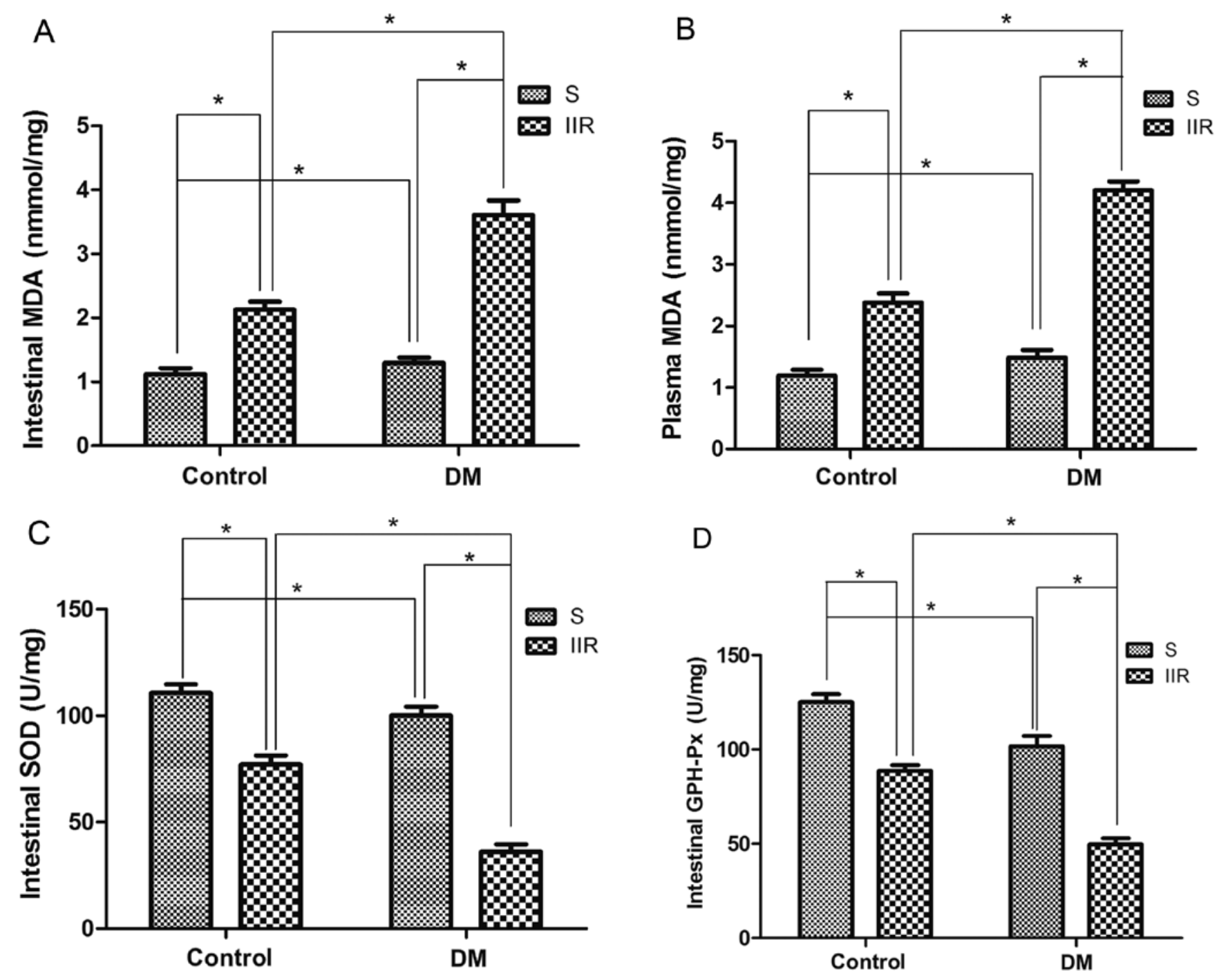

Figure 3. Diabetes influences oxidative stress during IIR. Levels of MDA in the (A) intestinal tissues and (B) serum were measured. Levels of (C) SOD and (D) GSH-Px activity in the intestinal tissues were measured. ${ }^{*} \mathrm{P}<0.05$, as indicated. IIR, intestinal ischaemia reperfusion; MDA, malondialdehyde; SOD, superoxide dismutase; GSH-Px, glutathione peroxidase; S, Sham; DM, diabetic mice.
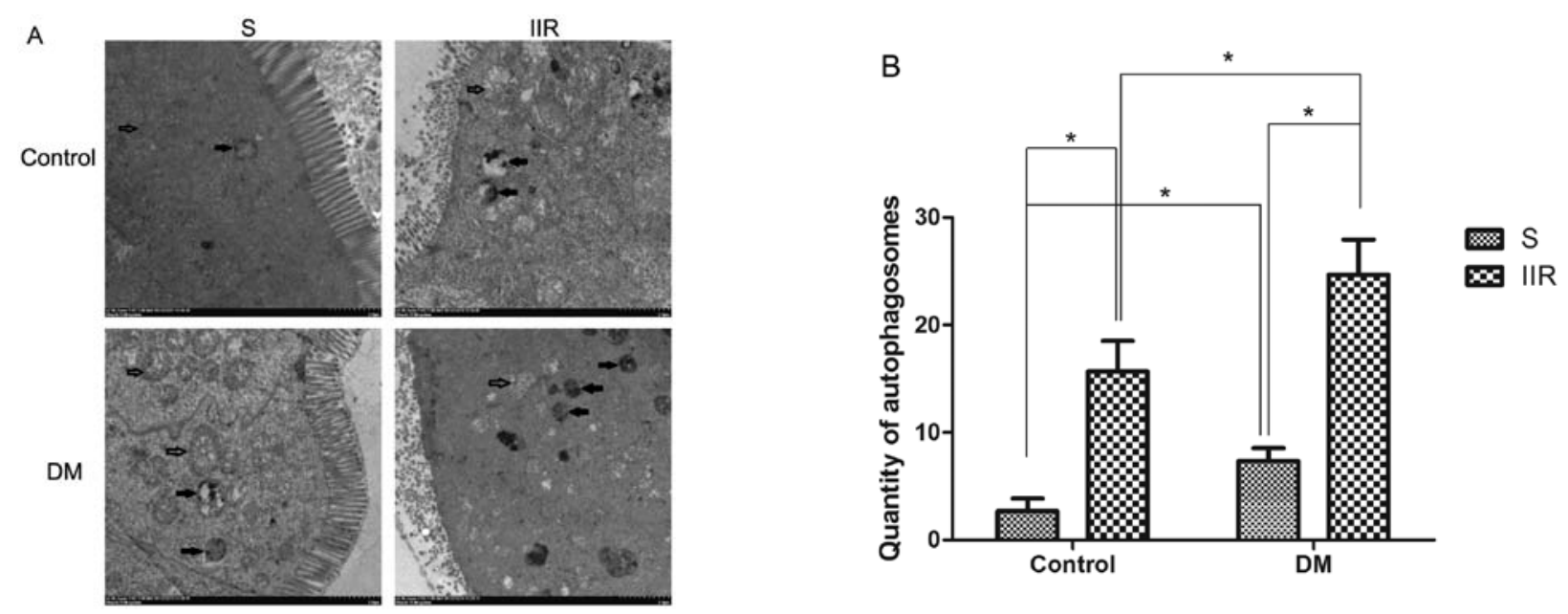

Figure 4. Effects of diabetes on IIR-induced intestinal. (A) Autophagosomes were observed under an electron microscope at $x 2,000$ magnification. (Solid arrows indicate mitochondria and hollow arrows indicate mitochondrial autophagy, scale bar $2.0 \mu \mathrm{m}$ ). (B) Histogram shows the average number of autophagosome structures per view $\left(371 \mu \mathrm{m}_{2}\right)$ obtained by examining at least 50 images per testing sample. Transmission electron microscopy indicated that the diabetic group displayed mitochondrial swelling, structural integrity and an increased number of autophagosomes compared with the Sham group. The IIR group displayed evident mitochondrial swelling, iliac crest rupture, decreased matrix density, vacuolated mitochondria and an increased number of autophagosomes compared with the Sham group. The DIIR group displayed a large number of destroyed mitochondria, undefined mitochondrial structure and an increased number of autophagosomes compared with the IIR group. ${ }^{*} \mathrm{P}<0.05$, as indicated. IIR, intestinal ischaemia reperfusion; DIIR, diabetes with IIR; S, Sham; DM, diabetic mice. 
A
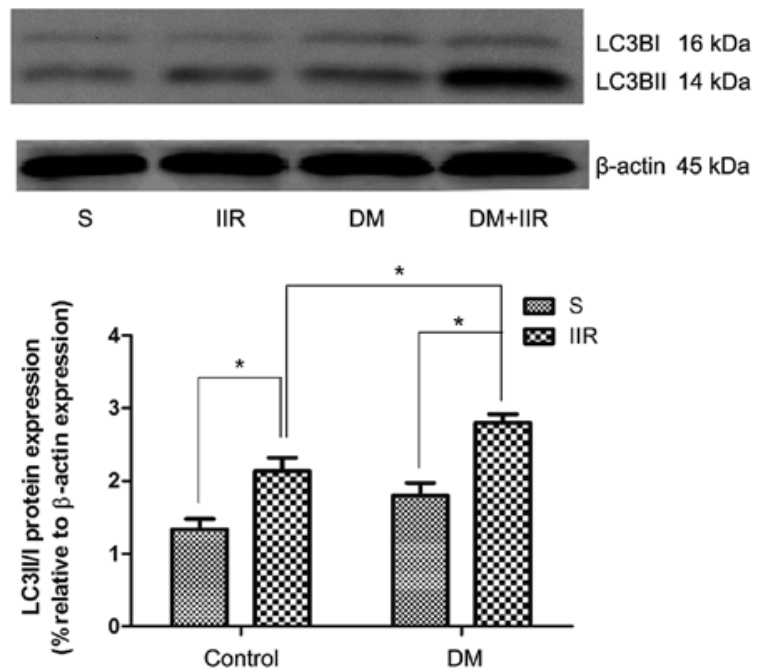

B
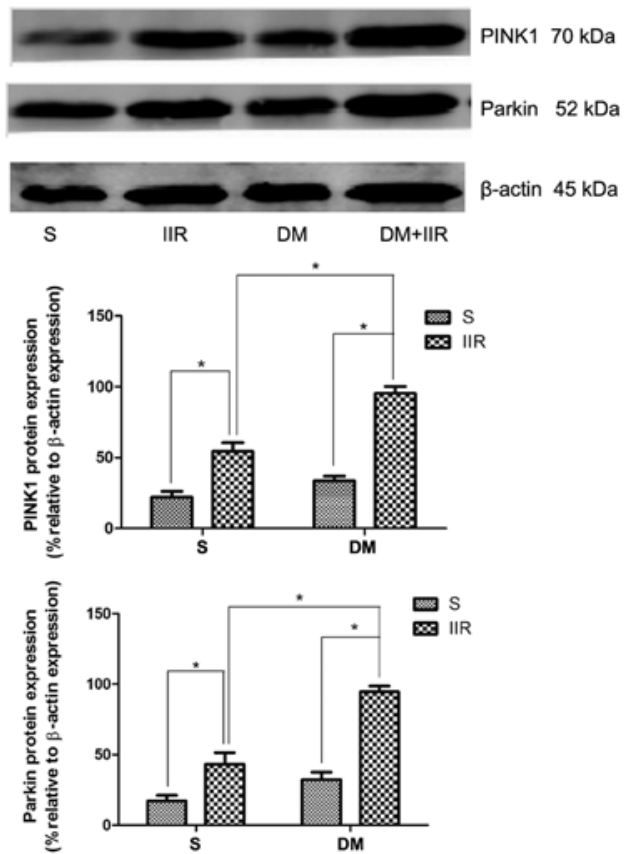

C

$\#$

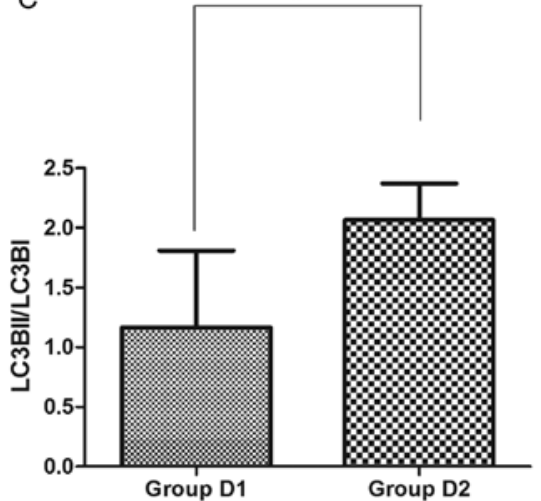

Figure 5. Protein expression levels of PINK1/Parkin and the ratio of LC3BII/I are upregulated following IIR in the mouse model of diabetes. Western blotting was performed to determine (A) the protein expression levels of PINK1 and Parkin, and (B) the ratio of LC3BII/I expression. ${ }^{*} \mathrm{P}<0.05$, as indicated. PINK1, phosphatase and tensin homolog-induced putative kinase; LC3BII/I, light chain 3B II/I; S, Sham; IIR, intestinal ischaemia reperfusion; DM, diabetic mice. (C) The difference in the ratio of LC3BII/I expression between the two group (DIIR group compared with the DS group vs. the IIR group compared with the Sham group). ${ }^{\#} \mathrm{P}<0.05$, as indicated. Group D1, difference between the IIR group and the Sham group; Group D2, difference between the DIIR group and the DS group. diabetes aggravates inflammatory reactions and oxidative stress, leading to catastrophic damage.

In the present study, the severity of pathological intestinal tissue damage was evaluated by H\&E staining combined with Chiu's score analysis. The results suggested that the intestinal mucosal structure, epithelial cell layer and lamina propria were severely destroyed, and the Chiu's score was significantly higher in the IIR groups compared with the corresponding control groups. The results indicated that IIR injury led to catastrophic damage to the intestinal tissue. The degree of intestinal mucosal damage in the DS and DIIR groups was more extensive compared with the Sham and IIR groups, respectively.

The mechanism that promotes the onset of IIR injury is multifactorial, complex and highly integrated. A number of biochemical pathways, including necrosis, apoptosis and autophagy, interact with one another $(38,39)$. In the present study, inflammation was significantly increased by upregulated expression levels of proinflammatory cytokines, including IL-1 $\beta$, IL- 6 and TNF- $\alpha$, in the DIIR group compared with the DS group. Following IIR in diabetic and non-diabetic mice, the level of MDA content increased, whereas the levels of SOD and GPH-Px activity decreased, suggesting that oxidative stress levels were significantly increased for all the experimental mice; however, the effects were more pronounced for the diabetic groups compared with the non-diabetic groups. The results indicated that following IIR, diabetes further increased inflammation and oxidative stress levels, and exacerbated intestinal tissue damage.

Furthermore, previous studies have reported that autophagy is closely related to inflammation and oxidative stress $(40,41)$. Zeng et al (42) demonstrated that the autophagy agonist rapamycin may reduce apoptosis by inhibiting excessive inflammatory responses and oxidative stress, thereby exerting a protective effect on intestinal tissue. Autophagy serves dual roles in a number of diseases via adaptive or maladaptive regulation. Physiological autophagy serves as a protective mechanism to maintain normal function; however, excessive autophagy contributes to disease development (43). In the present study, the western blotting results indicated that the extent of autophagy was significantly increased in the DIIR group compared with the IIR group. Furthermore, TEM experiments revealed that the mitochondrial structure of the DS group was notably damaged following IIR, and the basic outlines of the mitochondria became less clearly defined. Compared with the non-diabetic groups, mitochondrial destruction was more prevalent in the diabetic groups. The results of the western blot analysis revealed that the ratio of LC3BII/I was significantly higher in the diabetic groups compared with the non-diabetic groups, and these differences were more pronounced following IIR.

Autophagy is a key mechanism for maintaining normal cell function and stabilizing the internal environment for self-protection (44). Autophagy removes aging and damaged cells, and helps to eliminate pathogens, indicating an important role during normal development and in response to environmental stimuli (44). The mitochondrial kinase PINK1 and the E3-ubiquitin ligase Parkin, two Parkinson's disease-related proteins, function as the centers of mitochondrial quality control (45). PINK1 identifies damaged mitochondria and 
targets their degradation specifically via mitophagy (25). Following PINK1 accumulation on defective mitochondria, Parkin translocation from the cytosol is induced to mediate the clearance of damaged mitochondria by mitochondrial autophagy (46).

Increasing evidence has demonstrated that the activity of PINK1 is not restricted to mitophagy, but that different subcellular and submitochondrial pools of PINK1 are involved in distinct signaling cascades for the regulation of cell metabolism and survival (47). At the metabolic level, loss of PINK1 led to a significant inhibition of glucose uptake by pancreatic $\beta$-cells and primary intact islets, and was accompanied by increased insulin secretion and enhanced glucose tolerance (48). On this basis, the protein expression levels of PINK1 and Parkin were investigated in the present study. The results suggested that the expression of PINK1 and Parkin was significantly increased following IIR, and the observed increase was more pronounced in the diabetic groups compared with the non-diabetic groups. The results of the present study demonstrated that diabetes exacerbated the vulnerability to IIR, which was accompanied by alterations to autophagy. PINK1/Parkin may serve an important regulatory role during autophagy. Further studies are required to clarify the mechanisms underlying increased susceptibility to high-glucose-induced IIR by examining the effects of upregulation or inhibition of autophagy.

At present, intestinal ischemia is impossible to prevent and studies investigating IIR damage have been unsuccessful in translating the results of basic research into effective clinical prevention and treatment strategies. With the dramatic increase in patients with diabetes, a higher probability of undergoing surgery and suffering from IIR injury during the perioperative period has been observed (49-51). Further investigation into the pathological mechanisms underlying diabetes in IIR may help to identify novel preventative and therapeutic strategies, thereby improving survival.

To conclude, the present study indicated that diabetes aggravated intestinal damage following IIR in mice, and the increased vulnerability to IIR-induced intestinal damage due to diabetes may be related to PINK1/Parkin-regulated mitochondrial autophagy. However, the present study did not consider the detection of ROS using DCFH-DA; therefore, future studies employing this technique are required.

\section{Acknowledgements}

The authors would like to thank Dr Le Liu (Renmin Hospital of Wuhan University) for providing technical support for electron microscopy.

\section{Funding}

This study was supported by the National Natural Science Foundation of China (grant nos. 81671948 and 81401574).

\section{Availability of data and materials}

The datasets used and/or analyzed during the present study are available from the corresponding author on reasonable request.

\section{Authors' contributions}

QTM and ZYX designed the study. ZZ, YYZ, RC and TS performed the experiments. WL and HML provided vital reagents and analytical tools, edited and revised the manuscript. ZZ, WL, YYZ and QS performed the data analysis. ZZ, WL and HML wrote the manuscript. ZZ, HML and QTM prepared the illustrations and proofread the manuscript. All authors read and approved the final manuscript.

\section{Ethics approval and consent to participate}

The present study was approved by the Animal Care Committee of Renmin Hospital of Wuhan University and protocols were followed in accordance with the National Institutes of Health guidelines for the care and use of experimental animals.

\section{Patient consent for publication}

Not applicable.

\section{Competing interests}

The authors declare that they have no competing interests.

\section{References}

1. Miki T, Itoh T, Sunaga D and Miura T: Effects of diabetes on myocardial infarct size and cardioprotection by preconditioning and postconditioning. Cardiovasc Diabetol 11: 67, 2012.

2. Giacco F and Brownlee M: Oxidative stress and diabetic complications. Circ Res 107: 1058-1070, 2010.

3. D'Addio F and Fiorina P: Type 1 diabetes and dysfunctional intestinal homeostasis. Trends Endocrinol Metab 27: 493-503, 2016.

4. Nankervis CA, Giannone PJ and Reber KM: The neonatal intestinal vasculature: Contributing factors to necrotizing enterocolitis. Semin Perinatol 32: 83-91, 2008.

5. Yasuhara H: Acute mesenteric ischemia: The challenge of gastroenterology. Surg Today 35: 185-195, 2005.

6. Schwartz MZ: Novel therapies for the management of short bowel syndrome in children. Pediatr Surg Int 29: 967-974, 2013.

7. Posma LA, Bleichrodt RP, Lomme RM, de Man BM, van Goor H and Hendriks T: Early anastomotic repair in the rat intestine is affected by transient preoperative mesenteric ischemia. J Gastrointest Surg 13: 1099-1106, 2009.

8. Corcos O and Nuzzo A: Gastro-intestinal vascular emergencies. Best Pract Res Clin Gastroenterol 27: 709-725, 2013.

9. Fishman JE, Sheth SU, Levy G, Alli V, Lu Q, Xu D, Qin Y, Qin X and Deitch EA: Intraluminal nonbacterial intestinal components control gut and lung injury following trauma-hemorrhagic shock. Ann Surg 260: 1112-1120, 2014.

10. Sastry P, Hardman G, Page A, Parker R, Goddard M, Large S and Jenkins DP: Mesenteric ischaemia following cardiac surgery: The influence of intraoperative perfusion parameters. Interact Cardiovasc Thorac Surg 19: 419-424, 2014.

11. Hart ML, Gorzolla IC, Schittenhelm J, Dalton JH and Eltzschig HK: Retraction: Hypoxia-inducible factor-1 $\alpha$-dependent protection from intestinal ischemia/reperfusion injury involves Ecto-5'-nucleotidase (CD73) and the A2B adenosine receptor. J Immunol 199: 1942, 2017.

12. Puleo F, Arvanitakis M, Van Gossum A and Preiser JC: Gut failure in the ICU. Semin Respir Crit Care Med 32: 626-638, 2011.

13. Zhao W, Gan X, Su G, Wanling G, Li S, Hei Z, Yang C and Wang H: The interaction between oxidative stress and mast cell activation plays a role in acute lung injuries induced by intestinal ischemia-reperfusion. J Surg Res 187: 542-552, 2014.

14. Daniel RA, Cardoso VK, Góis JE Jr, Parra RS, Garcia SB, Rocha JJ and Féres O: Effect of hyperbaric oxygen therapy on the intestinal ischemia reperfusion injury. Acta Cir Bras 26: 463-469, 2011. 
15. Liu L, Tan Q, Hu B, Wu H, Wang C, Liu R and Tang C: Somatostatin Improved $\mathrm{B}$ cells mature in macaques during intestinal ischemia-reperfusion. PLoS One 10: e0133692, 2015.

16. Wu H, Deng YY, Liu L, Tan QH, Wang CH, Guo MM, Xie YM and Tang $\mathrm{CW}$ : Intestinal ischemia-reperfusion of macaques triggers a strong innate immune response. World J Gastroenterol 20 15327-15334, 2014.

17. Whittington HJ, Babu GG, Mocanu MM, Yellon DM and Hausenloy DJ: The diabetic heart: Too sweet for its own good? Cardiol Res Pract 2012: 845698, 2012.

18. Ansley DM and Baohua W: Oxidative stress and myocardial injury in the diabetic heart. J Pathol 229: 232-241, 2013.

19. Takayanagi R, Inoguchi $\mathrm{T}$ and Ohnaka K: Clinical and experimental evidence for oxidative stress as an exacerbating factor of diabetes mellitus. J Clin Biochem Nutr 48: 72-77, 2011.

20. Zhang P, Li T, Wu X, Nice EC, Huang C and Zhang Y: Oxidative stress and diabetes: Antioxidative strategies. Front Med: Apr 4, 2020 (Epub ahead of print)

21. Lenaz G, Bovina C, D'Aurelio M, Fato R, Formiggini G, Genova ML, Giuliano G, Merlo Pich M, Paolucci U, Parenti Castelli G and Ventura B: Role of mitochondria in oxidative stress and aging. Ann NY Acad Sci 959: 199-213, 2002.

22. Zorov DB, Juhaszova $M$ and Sollott SJ: Mitochondrial ROS-induced ROS release: An update and review. Biochim Biophys Acta 1757: 509-517, 2006

23. Tumurkhuu G, Koide N, Dagvadorj J, Morikawa A, Hassan F, Islam S, Naiki Y, Mori I, Yoshida T and Yokochi T: The mechanism of development of acute lung injury in lethal endotoxic shock using alpha-galactosylceramide sensitization. Clin Exp Immunol 152: 182-191, 2008 .

24. Shen X, Zheng S, Metreveli NS and Epstein PN: Protection of cardiac mitochondria by overexpression of MnSOD reduces diabetic cardiomyopathy. Diabetes 55: 798-805, 2006.

25. Sack MN: Type 2 diabetes, mitochondrial biology and the heart J Mol Cell Cardiol 46: 842-849, 2009.

26. Brownlee M: Biochemistry and molecular cell biology of diabetic complications. Nature 414: 813-820, 2001.

27. Wang S, Wang C, Yan F, Wang T, He Y, Li H, Xia Z and Zhang Z $\mathrm{N}$-Acetylcysteine attenuates diabetic myocardial ischemia reperfusion injury through inhibiting excessive autophagy. Mediators Inflamm 2017: 9257291, 2017.

28. International C: Guide for the care and use of laboratory animals Publication 327: 963-965, 2011.

29. Meng QT, Cao C, Wu Y, Liu HM, Li W, Sun Q, Chen R, Xiao YG Tang LH, Jiang Y, et al: Ischemic post-conditioning attenuates acute lung injury induced by intestinal ischemia-reperfusion in mice: Role of Nrf2. Lab Invest 96: 1087-1104, 2016.

30. Yu C, Tan S, Zhou C, Zhu C, Kang X, Liu S, Zhao S, Fan S, Yu Z, Peng $A$ and Wang Z: Berberine reduces uremia-associated intestinal mucosal barrier damage. Biol Pharm Bull 39: 1787-1792, 2016

31. Misra HP and Fridovich I: The role of superoxide anion in the autoxidation of epinephrine and simple assay for superoxide dismutase. J Biol Chem 247: 3170-3175, 1972.

32. Crane JD, Devries MC, Safdar A, Hamadeh MJ and Tarnopolsky MA: The effect of aging on human skeletal muscle mitochondrial and intramyocellular lipid ultrastructure. J Gerontol A Biol Sci Med Sci 65: 119-128, 2010.

33. Swank GM and Deitch EA: Role of the gut in multiple organ failure: Bacterial translocation and permeability changes. World J Surg 20: 411-417, 1996.

34. Gonzalez LM, Moeser AJ and Blikslager AT: Animal models of ischemia-reperfusion-induced intestinal injury: Progress and promise for translational research. Am J Physiol Gastrointest Liver Physiol 308: G63-G75, 2015.

35. Simos Y, Karkabounas S, Verginadis I, Charalampidis P, Filiou D, Charalabopoulos K, Zioris I, Kalfakakou V and Evangellou A: Intra-peritoneal application of catechins and EGCG as in vivo inhibitors of ozone-induced oxidative stress. Phytomedicine 18 $579-585,2011$.
36. Zu G, Guo J, Che N, Zhou T, Zhang X, Wang G, Ji A and Tian X: Protective effects of ginsenoside Rg1 on intestinal ischemia/reperfusion injury-induced oxidative stress and apoptosis via activation of the Wnt/ $\beta$-catenin pathway. Sci Rep 6: 38480 , 2016.

37. Nishikawa T, Edelstein D, Du XL, Yamagishi S, Matsumura T, Kaneda Y, Yorek MA, Beebe D, Oates PJ, Hammes HP, et al: Normalizing mitochondrial superoxide production blocks three pathways of hyperglycaemic damage. Nature 404: 787-790, 2000.

38. Wen Z, Hou W, Wu W, Zhao Y, Dong X, Bai X, Peng L and Song L: 6'-O-Galloylpaeoniflorin attenuates cerebral ischemia reperfusion-induced neuroinflammation and oxidative stress via PI3K/Akt/Nrf2 Activation. Oxid Med Cell Longev 2018: 8678267, 2018.

39. Wu H, Ye M, Yang J and Ding J: Modulating endoplasmic reticulum stress to alleviate myocardial ischemia and reperfusion injury from basic research to clinical practice: A long way to go. Int J Cardiol 223: 630-631, 2016.

40. Tsai YG, Wen YS, Wang JY, Yang KD, Sun HL, Liou JH and Lin CY: Complement regulatory protein CD46 induces autophagy against oxidative stress-mediated apoptosis in normal and asthmatic airway epithelium. Sci Rep 8: 12973, 2018.

41. Zhou M, Xu W, Wang J, Yan J, Shi Y, Zhang C, Ge W, Wu J, Du $\mathrm{P}$ and Chen Y: Boosting mTOR-dependent autophagy via upstream TLR4-MyD88-MAPK signalling and downstream $\mathrm{NF}-\kappa \mathrm{B}$ pathway quenches intestinal inflammation and oxidative stress injury. EBioMedicine 35: 345-360, 2018.

42. Zeng Z, Zhang YY, Tao S, et al: Protective effects of rapamycin on intestinal injury after intestinal ischemia-reperfusion in mouse. Med J Wuhan Univ 40: 192-196, 2019.

43. Jin S, Wei J, You L, Liu H and Qian W: Autophagy regulation and its dual role in blood cancers: A novel target for therapeutic development (Review). Oncol Rep 39: 2473-2481, 2018.

44. Klionsky DJ and Emr SD: Autophagy as a regulated pathway of cellular degradation. Science 290: 1717-1721, 2000.

45. Pickrell AM and Youle RJ: The Roles of PINK1, Parkin, and mitochondrial fidelity in Parkinson's disease. Neuron 85: 257-273, 2015.

46. Durcan TM and Fon EA: The three 'P's of mitophagy: PARKIN, PINK1, and post-translational modifications. Genes Dev 29: 989-999, 2015

47. Arena G and Valente EM: PINK1 in the limelight: Multiple functions of an eclectic protein in human health and disease. J Pathol 241: 251-263, 2017.

48. Deas E, Piipari K, Machhada A, Li A, Gutierrez-del-Arroyo A, Withers DJ, Wood NW and Abramov AY: PINK1 deficiency in $\beta$-cells increases basal insulin secretion and improves glucose tolerance in mice. Open Biol 4: 140051, 2014.

49. Rosenberg CS: Wound healing in the patient with diabetes mellitus. Nurse Clin North Am 25: 247-253, 1990.

50. Scannel G, Waxman K, Vaziri ND, Zhang J, Kaupke CJ, Jalali M and Hecht CC: Hypoxia-induced alterations of neutrophil membrane receptors. J Surg Res 59: 141-145, 1995

51. Thomaz Neto FJ, Koike MK, Abrahão Mde S, Carillo Neto F, Pereira RK, Machado JL and Montero EF: Ischemic preconditioning attenuates remote pulmonary inflammatory infiltration of diabetic rats with an intestinal and hepatic ischemia-reperfusion injury. Acta Cir Bras 28: 174-178, 2013.

This work is licensed under a Creative Commons Attribution-NonCommercial-NoDerivatives 4.0 International (CC BY-NC-ND 4.0) License. 